\title{
SEAM CARVING BASED IMAGE RESIZING DETECTION USING HYBRID FEATURES
}

\author{
Zehra Karapinar Senturk, Devrim Akgun
}

Original scientific paper

Detection of seam carving-based digital image resizing is a challenging task in image processing field since the method investigates the images on hand semantically. Resizing with seam carving is realized by inserting or removing relatively unimportant pixel paths to/from the images and so the changes in image content are mostly unnoticeable. Local Binary Patterns (LBP), a visual descriptor, unearths local changes in image texture. Therefore, using LBP transform of the images besides intensity values contributes to the detection ratio. In this paper, we proposed a hybrid detection mechanism for more accurate seam carving detection especially in low scaling ratios. We extracted LBP-based and non-LBP based features and trained a Support Vector Machine (SVM) with sixty features. We achieved approximately $9 \%$ improvement in low detection ratios. The experimental results show that more satisfactory detection ratios can be obtained by the proposed hybrid approach.

Keywords: forgery detection; Local Binary Patterns; seam carving; Support Vector Machines

\section{Otkrivanje promjene veličine slike temeljeno na tekućem skaliranju uporabom hibridnih značajki}

Izvorni znanstveni članak Otkrivanje promjene veličine digitalnih slika temeljeno na tekućem skaliranju je izazovna zadaća u području obrade slike budući da metoda istražuje slike na slici semantički. Promjena veličine s tekućim skaliranjem ostvaruje se umetanjem na slike ili uklanjanjem sa slika relativno nevažnih puteva piksela, zbog čega se promjene u slikovnom sadržaju uglavnom ne mogu primijetiti. Lokalni binarni uzorci (LBP), vizualni deskriptor, otkrivaju lokalne promjene u teksturi slike. Stoga, uporabom LBP transformacije slika osim vrijednosti intenziteta doprinosi se omjeru otkrivanja. U ovom smo radu predložili hibridni mehanizam otkrivanja za točnije otkrivanje tekućeg skaliranja, posebice u niskim omjerima skaliranja. Izdvojili smo značajke temeljene 1 netemeljene na LBP-u te sa šezdeset značajki vježbali podrškom vektorskih strojeva (SVM). Postigli smo približno $9 \%$ poboljšanja u omjerima niskog otkrivanja. Pokusni rezultati pokazuju da se zadovoljavajući omjeri otkrivanja mogu dobiti predloženim hibridnim pristupom.

Ključne riječi: lokalni binarni uzorci; otkrivanje krivotvorenja; podrška vektorskih strojeva; tekuće skaliranje (seam carving)

\section{Introduction}

Transforming images to their new sizes is a basic and a useful tool for many image processing operations. Saving image content and important foreground objects inside an image while resizing it, is one of the most popular research topics in image processing subject. Traditional resizing operations such as cropping and scaling are not satisfactory since they cause data losses (cropping) and visible distortions (scaling) in image content. Therefore, researchers started to search for content-aware resizing methods recently. Avidan and Shamir proposed an impressing approach for this purpose [1]. Their suggestion is to remove the least important pixel paths one by one to reduce image size or similarly to add new pixel paths which are placed in between two less important pixel paths. This approach eliminates most of the disadvantages of resizing operation and provides better visual quality.

Digital image forensics has been prevalent since the last decade. It aims to detect manipulations realized on the images. As the detection techniques are improved, more difficult forgery methods are developed by the falsifiers. Seam carving not only does content aware image resizing, but it can also remove objects in a given image. Since it handles the image in a semantic manner, it becomes more difficult to detect the forgery. Because the low-energy seams are removed from the image, the modifications are unnoticeable to human eyes.

Feature extraction and SVM (Support Vector Machines) have been frequently used in many fields of research such as energy management systems [2], watermarking [3], disease diagnosis [4, 5], industrial materials [6, 7], image [8] and signal processing [9] etc.
Also for the detection of seam carving, feature extraction and SVM based detection method are considered at first. First seam carving detection efforts have started two years later than the seam carving algorithm was proposed. It was the paper of Sarkar et al. [10] that investigates seam carving in terms of forgery detection. They developed a machine learning based seam carving detection method. Classification of whether an image is seam carved or seam inserted is realized thanks to 324 dimensional Markov feature and 2D difference histograms in DCT (Discrete Cosine Transform) domain. Features are trained with an SVM (Support Vector Machine). Seam carving and seam insertion are detected $80 \%$ and $85 \%$ respectively. It is assumed for seam insertion detection that every new pixel is a linear combination of its neighbors and high detection ratio (94\%) is obtained with low insertion rates. For seam insertion detection, neighborhood relations have been benefited. When the image is enlarged by seam carving, intermediate pixel path is placed between the seam path and one of its neighbors. Therefore, the change in the values of left, right, upper, and lower neighbor pixels reveal seam insertion. They considered the effect of smoothness, noise, and compression attacks for detection of seam carving since they may cause seam-like values in the calculation based on neighbor relations. They tried to reduce false detections caused by smooth regions by ignoring columns having no edge inside or the distance between the consequent edges far enough from each other after the edges are extracted by Canny filter. Seam carving detection is realized by the help of Markov process. Horizontal, vertical, main diagonal and minor diagonal difference arrays are modelled using Markov random process. Four transition probability matrices are 
established from four difference arrays. These four matrices are used as features to train an SVM (Support Vector Machine). Fillion and Sharma [11] proposed a pattern recognition approach and they considered four groups of features to train a Support Vector Machine. All feature vectors are calculated based on the gradient histogram of the image. They grouped features as energy based, seam behavior based, higher order statistics based (Wavelet Absolute Moments), and second seam carve based. First feature vector of energy based features is composed of the percent of pixels which are less than the predefined energy threshold. Second and third feature vectors are composed of mid-level and higher level histogram values respectively. Last group of features is obtained from CDF (Cumulative Distribution Function). Normalized CDF is used for better separation. Seam behavior based features are distance between the optimal seams at specific points and cumulative energy there. They plotted the graph which indicates optimal seams which passes through $10 \%, 25 \%, 50 \%$, and $100 \%$ of the original and seam carved image. They also showed cumulative energies at those points. From the graphs given in the paper, it is obvious that seams in the original image show more continuous form, but in seam carved image, seams are scattered, i.e. there is much distance between seams. They also claimed that seam carving influences the statistics of the image and they used wavelet absolute moment features, too. Lastly, they applied seam carving to the current image and extracted all previously mentioned features for the carved image. They showed that their features are quite effective for benign image resizing, but not in malignant object removal. They concluded that there are many factors which affect the detection accuracy of detection of object removal such as object size, object location, and directionality of the reduction [11].

In another paper, seam carving is tried to be detected with the help of side information, forensic hash, attached to the image and it is based on visual words representation of SIFT (Scale Invariant Feature Transform) [12]. This method not only decides whether the image is seam carved or not but it also estimates the location and the amount of seam carving. SIFT points are found in the original and tempered images and they are compared. The distances between two consecutive SIFT points in the original and the tempered images are calculated. The difference of the distances gives clue whether the image is seam carved or inserted. Positive difference means that some seams are removed between the corresponding points and negative difference means that some seams are inserted there. 200 original images and 800 seam carved images are used for the experiments. Although this method achieves high detection accuracy (99.4\% average correct detection probability), it is not applicable for many cases since it is not a blind technique. It requires information about the original image to compute and compare SIFT points.

Ryu et al. proposed a forensic technique to disclose seam carving by exploiting features of seam carving process [13]. Seam carving eliminates low energy pixel paths from the image, and so, the average remaining energy of the image increases. They extract fourteen statistical features (average column energy, average row energy, average energy, average energy difference, (for both row and column) seam max, seam min, seam mean, seam standard deviation, seam difference) based on the energy values of the images and used those features to train an SVM (Support Vector Machine). To detect seam addition, they used correlation between adjacent pixels. They established a database as a combination of the original images from UCID [14] and the carved images. They totally used 8028 images during the experiments. Detection ratio of $50 \%$ seam carved images is found as 93.50. A different approach for seam carving detection is proposed in a poster by Wei et al. [15]. A patch analysis is realized on the images. They divide the image into $2 \times 2$ small sub images, mini squares, and establish nine patches in accordance with these mini squares. They also used a referee pattern which decides the most suitable patch based on cosine similarity. The patches are constructed by interpolation between neighbors. After constructing patches, they constituted patch transition probability matrices which connect mini squares in vertical, sub diagonal and diagonal directions. 252-dimensional feature vector that includes probabilities of nine patches and the entries of transition probability matrices is established. An SVM is trained with these feature vectors including both carved and non-carved images. The detection accuracies of the method are $92.2 \%, 92.6 \%$, and $95.8 \%$ for $20 \%$, $30 \%$, and $50 \%$ carved images. Ryu et al. extended their work in [16]. They also considered noise in the images and added four more features related to noise. Noise of an image is computed by Wiener filter with $5 \times 5$ window and mean, standard deviation, skewness, and kurtosis are calculated based on the noise image. Experimental procedure is the same as what they did before. 1338 original, 6690 seam carved and 8028 seam inserted images are used in the experiments. They showed that their proposal outperforms [10] and [11] with detection ratio 93.57 vs 84.23 [10] and 71.90 [11]. Wei et al. elaborated their poster work in a paper [17]. They gave the details of the patch analysis method and compared the results of seam carving using forward energy and backward energy. They showed that seam carving which uses forward energy is more detectable than the one with backward energy. The reason of this is related to wide distribution of seams in forward-energy one. They also evaluated the transition probability matrices of the patches and generalized that "optimal patches of the mini squares tend to concentrate into just three of nine types, namely, types 0, 4, and 8". They also tried Euclidean distance as similarity metric and analyzed hot regions in the image. Mini squares are scored and low-score mini squares are ignored. Scoring is done based on transition probabilities of the patches. High-score mini squares imply hot regions, i.e. modified areas, in the image. Object removal is left as a future plan in this paper.

Patch analysis is applied to detect seam inserted images in [18]. The methodology is the same as [15]. Instead of cosine similarity metric in the comparison of the patches with referee pattern, they used Euclidean distance. Accuracies of the method are convincing with ratio more than $94 \%$.

All the previously mentioned detection methods work in pixel domain. Yin et al. found out that LBP (Local Binary Pattern) value of the neighboring pixels of a seam 
change significantly after seam carving, i.e. LBP value of a pixel betrays seam carving operation [19]. That is why they converted the image into LBP domain and then started detection of seam carving. They also saw that the optimal seam for an image is not optimal for half of the image. Therefore, they introduce half seam features which consider upper half of the images. Those features are min, max, and mean values based on cumulative minimum energy matrix of the half image in both column and row directions. Those six half seam features are combined with eighteen features proposed by [16] calculated based on LBP values and an SVM is trained with twenty-four features.

In this paper, a hybrid approach for more accurate seam carving detection has been proposed. The detection ratios have been increased by combining non-LBP based features of [16], LBP-based features of [19] and twelve new features. Totally, sixty features have been given as an input to a Support Vector Machine (SVM). The trained SVM has been used for the prediction of the images whose fates are unknown. The remainder of this paper is organized as follows: Section 2 gives background information about the method, Section 3 explains the proposed hybrid detection method, Section 4 presents experimental results, and lastly conclusions are made in Section 5.

\section{Background \\ 2.1 Image resizing with seam carving}

With the proliferation of digital image devices, resizing images, especially content-aware resizing is used more in smart phones, monitors, and HTML. Images can be enlarged/straightened to different sizes via scaling and even weight and height can be processed with different rates. But since this scaling operation is realized equally on every object of the image and since this will damage or will not reflect image content, it is not a good method. On the other hand, seam carving only adds/removes seam(s) which includes less information for enlarging/shrinking. Therefore, a content-aware resizing of images is performed with seam carving. A seam is an optimal 8connected path of pixels on a single image from top to bottom, or left to right, where optimality is defined by an image energy function [1]. Seam carving adds seams to the image for enlarging or to remove seams from image for lessening. As a result, this method removes the most negligible pixels and preserves fundamental features of the image and so, the alterations are inconceivable. The idea behind seam carving is to find unimportant pixel paths (seams) and remove them one by one until the desired size has been achieved. So, the important thing is to find the seams, i.e. optimal paths. Determination of the optimal path is realized via the energy function. Several energy functions including gradient magnitude can be used [1]. The algorithm for shrinking an image is given below.

Similar process is held for image enlargement. Instead of removing the optimal seam, an artificial pixel path which is composed of interpolation between optimal seam and its neighbors is added to the image. If the image width reduction or enlargement is intended, then vertical seams must be found and operated. Similarly, if the image height is to be changed, then horizontal seams will be found. For resizing in both directions, firstly, the order of the operation must be determined. This order may be important in terms of visual artifacts. In this case, adaptive determination of the order may eliminate artifacts.

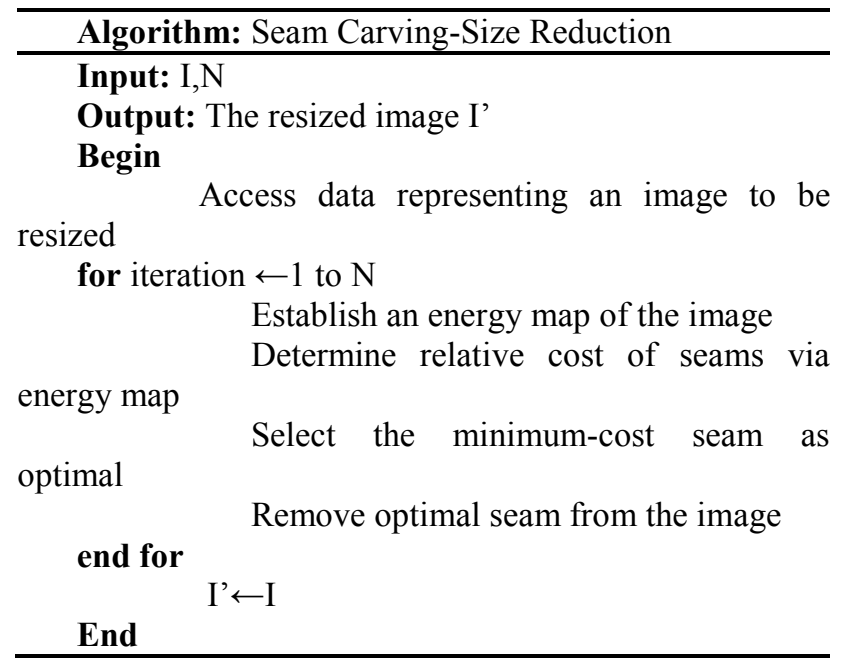

\begin{tabular}{|c|c|c|c|}
\multicolumn{5}{c}{ Pixel Costs } \\
\hline 46 & 25 & 12 & 95 \\
\hline 14 & 65 & 44 & 15 \\
\hline 18 & 22 & 56 & 22 \\
\hline 17 & 5 & 61 & 81 \\
\hline
\end{tabular}

\begin{tabular}{|c|c|c|c|}
\hline \multicolumn{4}{|c|}{ Minimum Costs } \\
\hline 46 & 25 & 12 & 95 \\
\hline $14+25$ & $65+12$ & $44+12$ & $15+12$ \\
\hline 18 & 22 & 56 & 22 \\
\hline 17 & 5 & 61 & 81 \\
\hline
\end{tabular}

\begin{tabular}{|c|c|c|c|}
\hline \multicolumn{5}{|c|}{$2^{\text {nd }}$ Row Iteration } \\
\hline 46 & 25 & 12 & 95 \\
\hline 39 & 77 & 56 & 727 \\
\hline $18+39$ & $22+39$ & $56+27$ & $22+27$ \\
\hline 17 & 5 & 61 & 81 \\
\hline
\end{tabular}

Finished Minimum Costs

Figure 1 Forming minimum cost table

\begin{tabular}{|c|c|c|c|}
\multicolumn{5}{c}{ Identify Minimum } \\
\hline 46 & 25 & 12 & 95 \\
\hline 39 & 77 & 56 & 27 \\
\hline 57 & 61 & 83 & 49 \\
\hline 74 & 62 & 110 & 130 \\
\hline
\end{tabular}

\begin{tabular}{|c|c|c|c|}
\hline 46 & 25 & 12 & 95 \\
\hline 39 & 77 & 56 & 27 \\
\hline 57 & 61 & 83 & 49 \\
\hline 74 & 62 & 110 & 130 \\
\hline
\end{tabular}

Figure 2 Determining minimum-energy (optimal) seam

Fig. 1 and 2 show optimal seam finding process. Minimum cost table is first established from top to bottom for vertical seam or from left to right for horizontal seam. A vertical seam is intended to be found in the figures. First row of the table is copied from the first row of the energy map and the following rows are formed by adding the least-value neighbor to current pixel value. Left, top and right neighbor values are compared and the value of the smallest one is added to current value. Since border pixels have only two upper neighbors, only top and left/right neighbors are compared to find their new corresponding value. Once the minimum cost table is established, the least-value pixel in this table is selected as the bottom pixel of the optimal seam and the pixels are stepped upwards based on their values. The place of the next step is decided among upper neighbors and the leastvalue pixel is selected until the first row is reached (see 
Fig. 2). An example image and its gradient with an optimal seam are shown in Fig. 3.

\subsection{Local binary patterns}

Local Binary Patterns, proposed in 1994, is a kind of visual descriptor to be used for classification purpose [20]. To obtain LBP form of an image, the image is first divided into cells and each of the neighboring pixels is compared with each pixel in a cell. The pixels are followed along a circle in clockwise or counter clockwise direction. If the value of the center pixel is greater than the value of the neighboring pixel, then 0 is written. If not, 1 is written. An 8-bit binary number is obtained in this way and it is converted to decimal for simplicity. This neighborhood distance may change. In this paper, we started comparing from the upper neighbor of the central pixel and follow the neighbors in clockwise direction. That is, the least significant digit of the eight bit number is the upper neighbor of the central pixel. Nearest neighbors have been regarded, i.e. the neighbors with distance one.

\subsection{Support Vector Machines (SVM)}

Support Vector Machine (SVM) is such a classification mechanism that fits a hyperplane between the classes. This hyperplane can separate even the classes which are not linearly separable with some modifications. Kernel functions are used to map those non-linearly separable classes of data into a space in which the data is linearly separable. SVMs maximize the margin between the closest data points of different classes. In this paper, SVM is used for the classification of seam carved and the original images. Several features are extracted and those features comprise the input of SVM. Since this is a twoclass classification problem, there will be two outputs: seam carved or original.

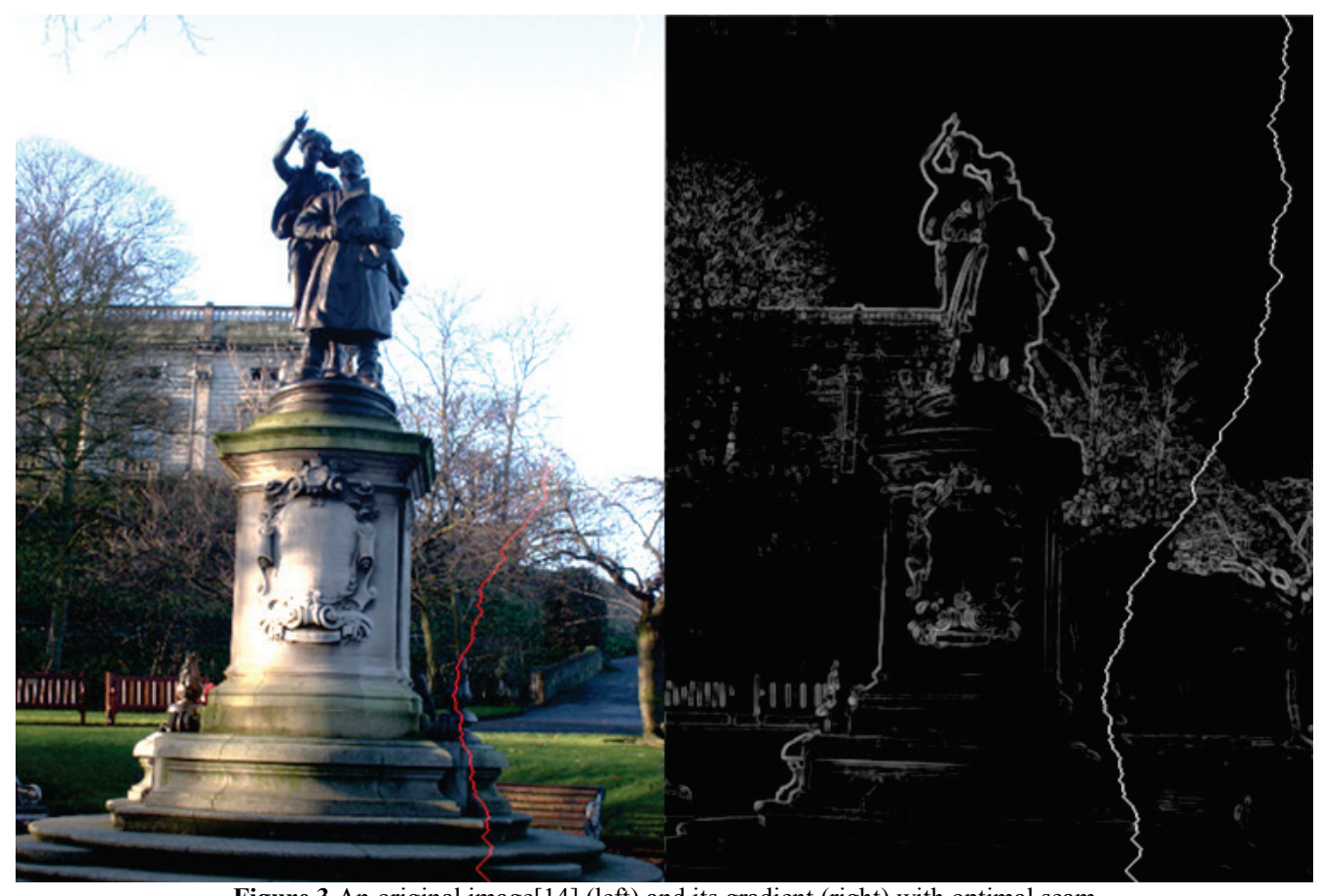

Figure 3 An original image[14] (left) and its gradient (right) with optimal seam

\section{Hybrid seam carving detection}

Removing one or more seams from an image increases average energy of the current image since the least important pixel(s) are removed. Therefore, analyzing image energy may contribute to detection operation. In detail, average energy of the remaining seams of the image will increase when a seam is removed. Besides, noise level of the image is also affected from seam carving because of removing usually flat regions [16]. These reasons direct to use eighteen features mentioned in [16]. On the other hand, since seam carving handles the images semantically, some common artifacts of image resizing like blurriness or shadows do not occur and the artifacts of seam carving can be in the local texture and around the removed or inserted seams. Therefore, a local texture descriptor brings to light the seam carving operation. If the features are extracted over the LBP transform of the images, then manipulations will be more apparent since local texture is emphasized with LBP transform. Although inter-pixel correlation and cooccurrence matrices have been used for that kind of artifacts, they are not effective for seam carving [19]. This idea leads to use aforementioned features in LBP domain. [19] adds also the features of the half image for the reason that the seams passing over the whole image and the upper half of the image do not overlap which means when the whole seam is carved there are deteriorations in the upper half of the image since that seam is not the optimal one for the upper half of the image. Therefore, analyzing upper half in terms of seam features may give clue about manipulations with seam carving. In the following section the features will be explained in detail.

The detection mechanism of the hybrid method works as follows: 
1. Establish training and testing data sets

2. Obtain corresponding LBP matrices for each image in the datasets

3. Extract 24 LBP based and 18 non-LBP based features for each LBP image

4. Extract 12 seam based features of the lower half image ( 6 in pixel domain, 6 in LBP domain).

5. Extract 6 seam based features of the upper half image in pixel domain.

6. Train an SVM with 60 features of the images in the training set

7. Use trained SVM to predict whether the images in the test set are seam carved or not using their features

Table 1 Energy-based features [16]

\begin{tabular}{|l|c|}
\hline \multicolumn{1}{|c|}{ Feature } & Description [16] \\
\hline Average row energy & $\frac{1}{m \times n} \sum_{i=1}^{m} \sum_{j=1}^{n}\left|\frac{\partial}{\partial y} I(i, j)\right|$ \\
\hline Average column energy & $\frac{1}{m \times n} \sum_{i=1}^{m} \sum_{j=1}^{n}\left|\frac{\partial}{\partial y} I(i, j)\right|$ \\
\hline Average energy & $\frac{1}{m \times n} \sum_{i=1}^{m} \sum_{j=1}^{n}\left(\left|\frac{\partial}{\partial x} I(i, j)\right|\right.$ \\
\hline $\begin{array}{l}\text { Average energy } \\
\text { difference }\end{array}$ & $\frac{1}{m \times n} \sum_{i=1}^{m} \sum_{j=1}^{n}\left(\left|\frac{\partial}{\partial x} I(i, j)\right|\right.$ \\
\hline Average row energy & $\frac{1}{m \times n} \sum_{i=1}^{m} \sum_{j=1}^{n}\left|\frac{\partial}{\partial y} I(i, j)\right|$ \\
\hline
\end{tabular}

Table 2 Seam-based features[16]

\begin{tabular}{|l|c|}
\multicolumn{1}{|c|}{ Table 2 Seam-based features[16] } \\
\hline Maximum & Description[16] \\
\hline Minimum & $\max _{i=1}^{m} C(i, n)$ \\
\hline Mean & $\frac{1}{m} \sum_{i=1}^{m} C(i, n)$ \\
\hline Standard Deviation & $\sqrt{\frac{1}{m} \sum_{i=1}^{m} \operatorname{Mean}-C(i, n)}$ \\
\hline Difference & $\max _{i=1}^{m} C(i, n)-\min _{i=1}^{m} C(i, n)$ \\
\hline
\end{tabular}

Table 3 Noise-based features[16]

\begin{tabular}{|l|c|}
\hline Feature & Description[16] \\
\hline Mean & $\frac{1}{m \times n} \sum_{i=1}^{m} \sum_{j=1}^{n} N(i, j)$ \\
\hline Standard Deviation & $\frac{1}{m \times n} \sum_{i=1}^{m} \sum_{j=1}^{n}(N(i, j)-\text { Mean })^{2}$ \\
\hline Skewness & $\frac{1}{m \times n} \sum_{i=1}^{m} \sum_{j=1}^{n}\left(\frac{N(i, j)-\text { Mean }}{\text { Standard Deviation }}\right)^{3}$ \\
\hline Kurtosis & $\frac{1}{m \times n} \sum_{i=1}^{m} \sum_{j=1}^{n}\left(\frac{N(i, j)-\text { Mean }}{\text { Standard Deviation }}\right)^{4}$ \\
\hline Mean & $\frac{1}{m \times n} \sum_{i=1}^{m} \sum_{j=1}^{n} N(i, j)$ \\
\hline
\end{tabular}

\subsection{Extracted features}

The features can be grouped as energy-based, seambased, noise-based, upper half seam-based, lower half seam-based. Some statistical features which define the images are intended to be used in SVM training. These features are to be extracted both in pixel domain and LBP domain. Formulations are given for an $m \times n$ image. Seam based features are calculated based on Cumulative Minimum Energy Matrix (C) and calculated both for row and column directions. Upper and lower half seam features are only minimum, maximum, and mean values of all possible seams in the upper or lower half of the images. Noise of the image $(\mathrm{N})$ has been extracted by the help of Wiener Filter with $5 \times 5$ window. Energy-based features, seam-based features and noise based features are summarized in Tab. 1-3.

\section{Experimental results \\ 4.1 Experimental setup}

In the experiments, the detector is run on Octave which is free and high level software designed especially for numerical calculations [21]. SVM classifier is downloaded from LibSVM [22]. Radial basis function is used as a kernel function. Its parameters $\mathrm{C}$ (cost) and $\gamma$ (Gamma) are determined by the help of grid search. The values of these parameters directly affect detection accuracy. Therefore, the best $\mathrm{c}$ and gamma have been selected. In addition, a database is established to be used in training and testing phases of the detection approach. For this purpose, 1338 images have been downloaded from UCID [14] and these original images have been scaled by seam carving with $10 \%, 20 \%$, and $30 \%$ scaling ratios. Scaling more than $30 \%$ is not considered since the modifications will be more visible; it can be seen even visually and requires no detailed analysis. There are 1338 original $+1338 \times 3$ tempered images (totally 5350 images) in the database for the experiments of the proposed detector. Vertical seam removal has been considered in all experiments, i.e. width reduction by seam carving is performed. For equal comparison with [19], the same scaling ratio is used in the training and testing images. For example, while detecting whether the image is scaled $30 \%$ with seam carving, we used $30 \%$ scaled images with their originals to train SVM. Four fold cross validation methodology is exploited. Three fourths of randomly selected images are used in training and the remaining one fourth of the images is used in testing. That is, there are 2006 images in the training set and 670 images in the test set for each scaling ratio. The measurements have been realized on a PC with Intel Core i7-4720HQ CPU@2.60 GHz and 16GB RAM.

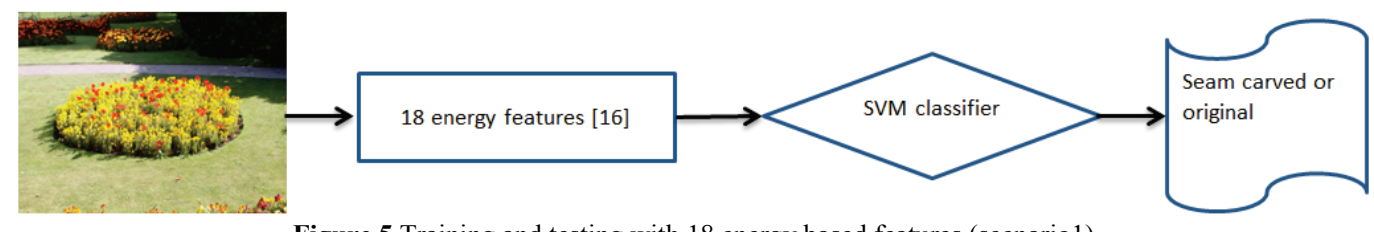

Figure 5 Training and testing with 18 energy based features (scenario1) 


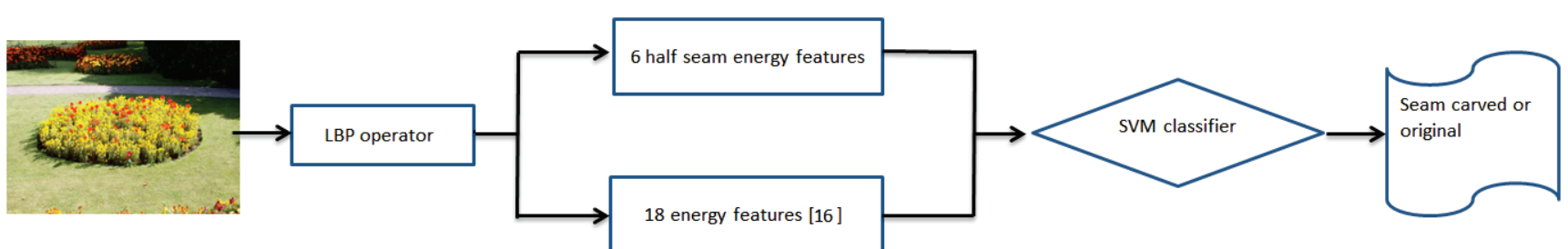

Figure 6 Training and testing with 24 LBP features [19] (scenario2)

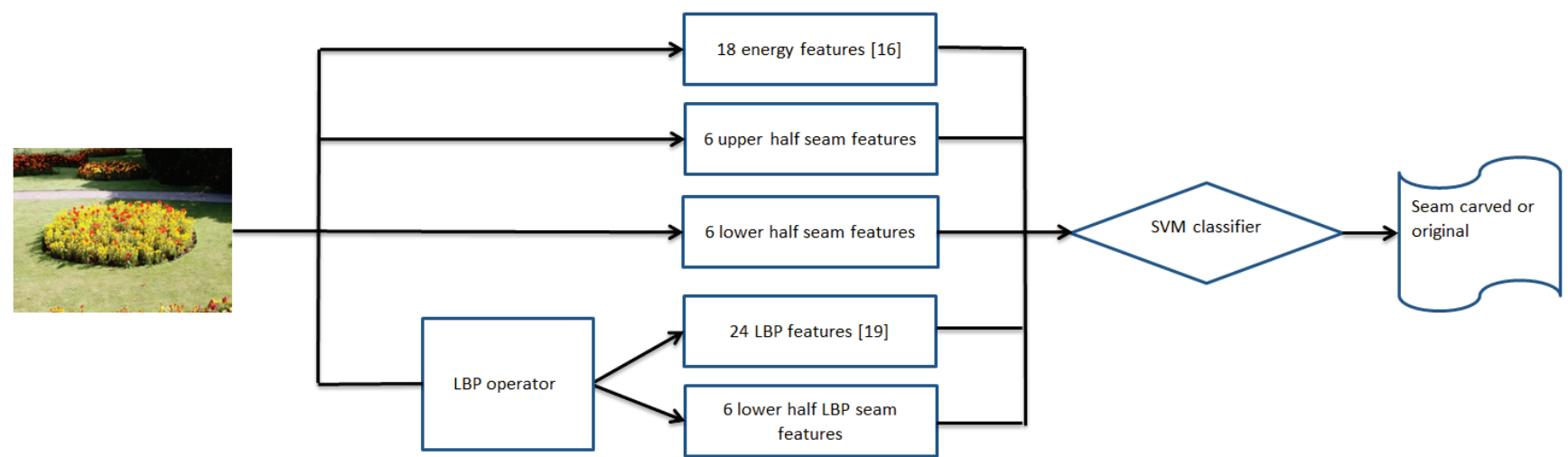

Figure 7 Training and testing with hybrid features (scenario3)

\subsection{Detection results for the scenarios}

Experiments have been conducted according to the scenarios given in Figs. 5-7. Shortly, first, the detection ratios of the method proposed in [16] have been obtained. Eighteen energy based features were given to an SVM and the average detection ratios after four fold cross validation were measured. Secondly, the effect of LBP transform is shown. After the images are transformed into LBP domain, all previously mentioned features of [16] and six new seam features of the upper half of the image have been extracted. Last but not least, the proposed method has been experimented. Six upper half seam features and six lower half seam features are added to eighteen energy-based features. Similarly, lower half features are added to LBP based features and these two feature sets are combined. This hybrid set contains sixty features including LBP-based and non-LBP based features. The detection performances of the proposed approach are recorded for each scaling ratio as given in Tab. 4-6.

Table 4 Detection Accuracies (\%)

\begin{tabular}{|c|c|c|c|}
\hline $\begin{array}{c}\text { Scaling Ratio } \\
(\%)\end{array}$ & $\begin{array}{c}\text { Energy-based } \\
{[16]}\end{array}$ & $\begin{array}{c}\text { LBP-based } \\
{[19]}\end{array}$ & $\begin{array}{c}\text { Proposed } \\
\text { Detector }\end{array}$ \\
\hline 3 & 53.25 & 54.26 & 61.85 \\
\hline 6 & 63.19 & 64.57 & 73.02 \\
\hline 10 & 71.53 & 81.73 & 85.69 \\
\hline 20 & 79.56 & 93.05 & 94.69 \\
\hline 30 & 86.40 & 96.26 & 97.65 \\
\hline
\end{tabular}

Table 5 False Positive Ratio (\%)

\begin{tabular}{|c|c|c|c|}
\hline $\begin{array}{c}\text { Scaling Ratio } \\
(\%)\end{array}$ & $\begin{array}{c}\text { Energy-based } \\
{[16]}\end{array}$ & $\begin{array}{c}\text { LBP-based } \\
{[19]}\end{array}$ & $\begin{array}{c}\text { Proposed } \\
\text { Detector }\end{array}$ \\
\hline 3 & 44.91 & 57.91 & 41.19 \\
\hline 6 & 33.23 & 45.07 & 31.34 \\
\hline 10 & 21.26 & 26.95 & 19.16 \\
\hline 20 & 17.07 & 8.383 & 7.16 \\
\hline 30 & 10.75 & 5.090 & 3.88 \\
\hline
\end{tabular}

Table 6 False Negative Ratio (\%)

\begin{tabular}{|c|c|c|c|}
\hline $\begin{array}{c}\text { Scaling Ratio } \\
(\%)\end{array}$ & $\begin{array}{c}\text { Energy-based } \\
{[16]}\end{array}$ & $\begin{array}{c}\text { LBP-based } \\
{[19]}\end{array}$ & $\begin{array}{c}\text { Proposed } \\
\text { Detector }\end{array}$ \\
\hline 3 & 49.10 & 31.44 & 29.55 \\
\hline 6 & 41.02 & 28.14 & 21.86 \\
\hline 10 & 30.24 & 13.47 & 9.55 \\
\hline 20 & 18.51 & 8.68 & 3.28 \\
\hline 30 & 16.42 & 2.69 & 1.79 \\
\hline
\end{tabular}

\subsection{Discussion}

LBP based seam carving detection method has been improved by some additional features. Proposed method presents a hybrid approach as a combination of LBP based and non-LBP based features. The reasons of the improvement are as follows. Adding upper and lower half seam features to energy image in pixel domain extracts the changes in energy level after seam carving. Since seam carving removes the lowest-energy pixel paths, the overall energy of the image increases. The logic behind looking at the upper and lower halves of the images is the same as [19], i.e. when a seam is found over an image, this will mostly be different than that found over the upper half of the image. These two seams mostly do not overlap. That is, the optimal seam of the image will not be the optimal one for the upper part of the image. Therefore, a pixel path which is not the lowest-energy one will be removed from the upper part. This will damage energy distribution of the image and the features found over here will unveil forgeries. Since a vertical seam can be found as not only top-down but it can also be found from bottom to top of the image. Therefore, analyzing lower half of the image besides the upper half increases detection ratio. As seen in Table 4, the effect of the newly proposed features stand out in the images which are scaled by low scaling ratios $(3 \%$ and $6 \%)$. Less amount of seam carving means higher average energy. Hence, the features related to the energy of the image will be higher in the images which are scaled in small scaling ratios. Also, the possible seams in those images will carry more energy and this means 
higher values in seam features. Besides, since more flat region will be removed in high scaling ratios, the noise level will be affected and so the features related to the noise of the image will reflect this situation. The effect of noise features will be more apparent in high scaling ratios. But energy based and seam based features suppress the effect of noise features.

Tab. 5 and Tab. 6 show false positive ratios and false negative ratios respectively. As seen in Tab. 6, false positive ratios and false negative ratios obtained by the proposed detector are considerably lower than those of LBP-based and energy-based approaches. This means that the overall number of the misclassified images (the number of the original images which are classified as seam carved and the number of the seam carved images which are classified as original) is highly less than the previous methods.

\section{Conclusions and future work}

In this paper, a very popular content aware image resizing technique, seam carving, is investigated in terms of forgery detection. When compared with other resizing operations, it is much more difficult to detect seam carving since it evaluates the images in a semantic way. It assigns importance value to every pixel and removes the pixel path with the lowest cumulative importance (energy) and so the resizing occurs mostly with few or no visual deterioration. Although it is not experimented in this paper, seam carving can be used maliciously to remove objects inside the images. The detection of object removal requires more comprehensive evaluation. It is more difficult than deciding whether the image is resized and it is left as a future plan. In this study, detection ratio of LBP based method which is the most successful seam carving detection method as far as we know is improved. LBP based features are combined with non-LBP based features of [16]. Also, lower half of the image is evaluated in terms of seam features and twelve additional features have been used. As a result, detection ratios are increased by the proposed sixty features. The difficulty of the proposed approach is the time required for feature extraction and feature elimination will be worked in the future. Determining the relatively important features and using only them for SVM training/testing will save time providing that the satisfactory detection ratios are obtained

\section{Acknowledgements}

This work was supported by Research Fund of the Sakarya University. Project Number: 2015-50-02-019

\section{References}

[1] Avidan, S.; Shamir, A. Seam carving for content-aware image resizing. // ACM Trans. Graph. 26, 3(2007), p. 10. https://doi.org/10.1145/1276377.1276390

[2] Wang, F.; Zhen, Z.; Mi, Z.; Sun, H.; Su, S.; Yang, G. Solar irradiance feature extraction and support vector machines based weather status pattern recognition model for shortterm photovoltaic power forecasting. // Energy Build. 86, (2015), pp. 427-438. https://doi.org/10.1016/i.enbuild.2014.10.002

[3] Verma, V. S.; Jha, R. K.; Ojha, A. Digital watermark extraction using support vector machine with principal component analysis based feature reduction. // J. Vis. Commun. Image Represent. 31, (2015), pp. 75-85. https://doi.org/10.1016/j.jvcir.2015.06.001

[4] Yu, S.; Tan, K. K.; Sng, B. L.; Li, S.; Sia, A. T. H. Lumbar Ultrasound Image Feature Extraction and Classification with Support Vector Machine. // Ultrasound Med. Biol. 41, 10(2015), pp. 2677-2689. https://doi.org/10.1016/j.ultrasmedbio.2015.05.015

[5] Sady, C. C. R.; Ribeiro, A. L. P. Symbolic features and classification via support vector machine for predicting death in patients with Chagas disease. // Comput. Biol. Med. 70, (2016), pp. 220-227. https://doi.org/10.1016/j.compbiomed.2016.01.016

[6] Saidi, L.; Ben Ali, J.; Fnaiech, F. Application of higher order spectral features and support vector machines for bearing faults classification. // ISA Trans. 54, (2015), pp. 193-206. https://doi.org/10.1016/.isatra.2014.08.007

[7] Li, Y.; Xu, M.; Zhao, H.; Huang, W. Hierarchical fuzzy entropy and improved support vector machine based binary tree approach for rolling bearing fault diagnosis. // Mech. Mach. Theory. 98, (2016), pp. 114-132.

https://doi.org/10.1016/j.mechmachtheory.2015.11.010

[8] Azhar, R.; Tuwohingide, D.; Kamudi, D.; Sarimuddin; Suciati, N. Batik Image Classification Using SIFT Feature Extraction, Bag of Features and Support Vector Machine. // Procedia Comput. Sci. 72, (2015), pp. 24-30. https://doi.org/10.1016/j.procs.2015.12.101

[9] Wu, Q.; Mao, J. F.; Wei, C. F.; Fu, S.; Law, R.; Ding, L.; Yu, B. T.; Jia, B.; Yang, C. H. Hybrid BF-PSO and fuzzy support vector machine for diagnosis of fatigue status using EMG signal features. // Neurocomputing. 173, (2016), pp. 483-500. https://doi.org/10.1016/j.neucom.2015.06.002

[10] Sarkar, A.; Nataraj, L.; Manjunath, B. S. Detection of seam carving and localization of seam insertions in digital images. // in Proceedings of the 11th ACM workshop on Multimedia and security - MM\&Sec '09, 2009, p. 107.

[11] Fillion, C.; Sharma, G. Detecting Content Adaptive Scaling of Images for Forensic Applications. Media Forensics and Security II, edited by Nasir D. Memon, Jana Dittmann, Adnan M. Alattar, Edward J. Delp III, Proc. of SPIE-IS\&T Electronic Imaging, SPIE Vol. 7541, 75410Z C 2010 SPIEIS\&T $\cdot$ CCC code: $0277-786 X / 10 / \$ 18$

[12] Lu, W.; Wu, M. Seam carving estimation using forensic hash. // in Proceedings of the thirteenth ACM multimedia workshop on Multimedia and security - MM\&Sec '11, 2011, p. 9.

[13] Ryu, S. J.; Lee, H. Y.; Lee, H. K. Detection of ContentAware Image Resizing Using Seam Properties. // Appl. Mech. Mater. 284-287, (2013), pp. 3074-3078. https://doi.org/10.4028/www.scientific.net/AMM.284-287.3074

[14] Gerald Schaefer, M. S. UCID - An Uncompressed Colour Image Database. // Storage and Retrieval Methods and Applications for Multimedia 2004, edited by Minerva M. Yeung, Rainer W. Lienhart, Chung-Sheng Li, Proc. of SPIE-IS\&T Electronic Imaging, SPIE Vol. 5307 (C) 2004 SPIE and IS\&T $\cdot 0277-786 \mathrm{X} / 04 / \$ 15$

[15] Wei, J.-D.; Lin, Y.-J.; Wu, Y.-J.; Kang, L.-W. A patch analysis approach for seam-carved image detection. // ACM SIGGRAPH 2013 Posters on - SIGGRAPH '13, 2013, p. 1.

[16] Ryu, S.-J.; Lee, H.-Y.; Lee, H.-K. Detecting Trace of Seam Carving for Forensic Analysis. // IEICE Trans. Inf. Syst. E97.D, 5(2014), pp. 1304-1311.

[17] Wei, J.-D.; Lin, Y.-J.; Wu, Y.-J. A patch analysis method to detect seam carved images. // IEEE Transactions on Image Processing. 36, (2014), pp. 100-106. https://doi.org/10.1016/j.patrec.2013.09.026

[18] Jiang, P.-Y.; Cheng, H.-J.; Wei, J.-D. Using Patch Analysis Methods to Detect Images Tampered with Seam Insertion. // Proceedings of the International Conference on 
Electronics and Software Science, Takamatsu, Japan, 2015, pp. 187-190.

[19] Yin, T.; Yang, G.; Li, L.; Zhang, D.; Sun, X. Detecting seam carving based image resizing using local binary patterns. // Comput. Secur. 55, (2015), pp. 130-141.

https://doi.org/10.1016/j.cose.2015.09.003

[20] Pietikäinen, M. Local Binary Patterns. // Scholarpedia. 5, 3(2010), p. 9775 . https://doi.org/10.4249/scholarpedia.9775

[21] Eaton, J. W. About Octave. https://www.gnu.org/software/ octave/about.html

[22] Chang, C.-C.; Lin, C.-J. LIBSVM: A Library for Support Vector Machines. // ACM Transactions on Intelligent Systems and Technology (TIST). 2, 3(2011), Article No. 27. https://doi.org/10.1145/1961189.1961199

\section{Authors' addresses}

\section{Zehra Karapinar Senturk, PhD Student}

Duzce University, Faculty of Engineering,

Department of Computer Engineering,

Konuralp Campus, 81620, Duzce, Turkey

E-mail: zehrakarapinar@duzce.edu.tr

\section{Devrin Akgun, Assistant Professor}

Sakarya University, Faculty of Computer and Information

Sciences, Computer Engineering Department,

Esentepe Campus, 54187, Sakarya, Turkey

E-mail: dakgun@sakarya.edu.tr 